\title{
EXPRESIÓN DE CDX2 EN LESIONES DE VEJIGA URINARIA Y URETRA
}

\author{
J.A. ORTIZ-REY, I. ANTÓN BADIOLA, P. SAN MIGUEL FRAILE, C. ÁLVAREZ ÁLVAREZ, \\ B. IGLESIAS RODRÍGUEZ, E. ZUNGRI-TELO*
}

\author{
Servicio de Patología. *Servicio de Urología. Centro Médico POVISA. Vigo (Pontevedra). \\ Actas Urol Esp. 28 (2): 101-105, 2004
}

\section{RESUMEN}

\section{EXPRESIÓN DE CDX2 EN LESIONES DE VEJIGA URINARIA Y URETRA}

FUNDAMENTO: CDX1 Y CDX2 son factores de transcripción esenciales para el desarrollo y mantenimiento de la célula epitelial intestinal. Se ha descrito la expresión de CDX2 en el epitelio intestinal normal y metaplásico, y en adenocarcinomas originados en el mismo. Hemos analizado la expresión de dicho marcador en lesiones reactivas y tumorales de vejiga, uretra y uraco.

MÉTODO: Se determinó CDX2 mediante inmunohistoquímica sobre tejido incluido en parafina, con el método de estreptavidina marcada-biotina (LSAB2, Dako) utilizando un anticuerpo monoclonal (CDX288, BioGenex).

RESULTADOS: Se observó expresión de CDX2 en la cistitis glandularis de tipo intestinal, metaplasia intestinal de vejiga, adenocarcinoma vesical, carcinoma mucinoso urotelial de uretra prostática y carcinoma mucinoso de uraco. Fueron negativos para CDX2 el urotelio y epitelio glandular prostático normales, nidos de Von Brunn, cistitis glandularis típica, adenosis glandular y carcinoma transicional.

CONCLUSIONES: Aquellas lesiones, benignas y malignas, con morfología de célula entérica presentan positividad para CDX2. La expresión de este marcador no es, por tanto, órgano-específica y únicamente se relaciona con un fenotipo celular. La positividad para CDX2 en un adenocarcinoma puede ser compatible con un origen en el tracto urinario o en el uraco.

PALABRAS CLAVE: Proteína Cdx-2. Proteínas del homeodominio. Epitelio intestinal. Metaplasia. Vejiga. Uretra. Inmunohistoquímica.

\section{ABSTRACT}

EXPRESSION OF CDX2 IN LESIONS OF URINARY BLADDER AND URETHRA

BACKGROUND: CDX1 and CDX2 are transcription factors involved in the development and maintenance of the intestinal epithelial cell. Expression of CDX2 has been reported in normal and metaplastic intestinal epithelium, and in those adenocarcinomas with that cellular origin. We have analized the expression of this marker in reactive and tumoral lesions arising in urinary bladder, urethra and urachus.

METHOD: CDX2 was investigated through immunohistochemistry on paraffin-embedded tissue, using the labelled streptavidin-biotin method (LSAB2, Dako) with a monoclonal antibody (CDX2-88, BioGenex).

RESULTS: Expression of CDX2 was observed in intestinal-type cistitis glandularis, intestinal metaplasia of urinary bladder, bladder adenocarcinoma, mucinous urothelial-type carcinoma of prostatic urethra and urachal mucinous carcinoma. CDX2 was not detected in normal urothelium and prostatic glandular epithelium, Von Brunn nests, typical-type cistitis glandularis, glandular adenosis and transitional carcinoma.

CONCLUSIONS: Lesions, both benign and malignant, with enteric-cell morphological features show positivity for CDX2. Expression of this marker is not organ-specific but is just related to a cellular phenotype. Reactivity for CDX2 in an adenocarcinoma can be consistent with an origin in urinary tract or urachus. 
$\mathrm{L}$ as proteínas CDX1 y CDX2 son factores de transcripción que promueven la diferenciación y el mantenimiento del epitelio intestinal normal $^{1}$. Se localizan en el núcleo celular y son claves no sólo para el desarrollo del epitelio del intestino delgado y grueso en el embrión sino también para la conservación de dicho fenotipo celular en el individuo ya maduro, en el periodo post-natal ${ }^{1}$.

Las concentraciones de CDX1 y CDX2 varían dependiendo del estadio de desarrollo del individuo, de la región anatómica del intestino y del tipo celular en la mucosa ${ }^{1,2}$.

Se han relacionado alteraciones en la expresión de CDX1 y CDX2 con la aparición y progresión de tumores en el colon ${ }^{3-5}$. La mayor parte de los tumores intestinales mantienen la expresión de CDX2, por lo que se ha propuesto que la presencia de este marcador en un adenocarcinoma sirve, en general, como indicador de origen colónico ${ }^{6-11}$. Se ha descrito, además, la expresión de CDX2 en la metaplasia intestinal de la mucosa gástrica y, por consiguiente, en los adenocarcinomas de tipo intestinal del estómago $7,12,13$.

En el tracto urinario existen diferentes lesiones, tanto benignas como malignas, que están constituidas por un epitelio morfológicamente de tipo intestinal ${ }^{14-19}$. El objetivo de este estudio es analizar mediante técnica de inmunohistoquímica la expresión de CDX2 en casos representativos de estas entidades.

\section{MATERIAL Y MÉTODOS}

Se han seleccionado casos representativos de patología benigna y maligna del tracto urinario que se relacionan en la Tabla I.

\section{TABLA I}

CASOS ESTUDIADOS

\begin{tabular}{|c|c|}
\hline Lesiones benignas & Patología maligna \\
\hline Nidos de Von Brunn & Carcinoma transicional \\
\hline $\begin{array}{c}\text { Cistitis glandularis } \\
\text { tipica }\end{array}$ & Adenocarcinoma de vejiga \\
\hline $\begin{array}{c}\text { Cistitis glandularis tipo } \\
\text { intestinal }\end{array}$ & $\begin{array}{c}\text { Adenocarcinoma mucinoso } \\
\text { transicional de uretra }\end{array}$ \\
\hline Metaplasia intestinal & $\begin{array}{c}\text { Adenocarcinoma mucinoso } \\
\text { de uraco }\end{array}$ \\
\hline Adenosis glandular & \\
\hline
\end{tabular}

Sobre secciones fijadas en formol e incluidas en parafina de manera rutinaria, se realizó técnica de inmunohistoquímica siguiendo el método de estreptavidina marcada con peroxidasabiotina (LSAB2 ${ }^{\circledR}$, Dako), utilizando un anticuerpo monoclonal anti-CDX2 prediluido (Clon CDX2-88, BioGenex). El desenmascaramiento antigénico se realizó mediante incubación en tampón citrato a pH 6.0 en olla, con presión máxima durante 5 minutos. El cromógeno utilizado fue diaminobencidina. La técnica se realizó además sobre controles positivos y negativos adecuados.

Se analizó al microscopio la positividad nuclear (coloración marrón) valorándose además la intensidad y proporción de células reactivas.

\section{RESULTADOS}

El urotelio y el epitelio glandular prostático normales fueron negativos para CDX2.

En todas las lesiones con presencia de células con morfología de tipo intestinal, CDX2 fue positivo: se observó expresión focal y de intensidad discreta en la cistitis glandularis de tipo intestinal (Fig. 1); intensa y generalizada en la metaplasia intestinal de la vejiga (Fig. 2), adenocarcinoma de tipo intestinal de vejiga (Fig. 3) y carcinoma mucinoso de tipo urotelial de uretra; y positividad moderada y generalizada en un carcinoma mucinoso de uraco (Fig. 4).

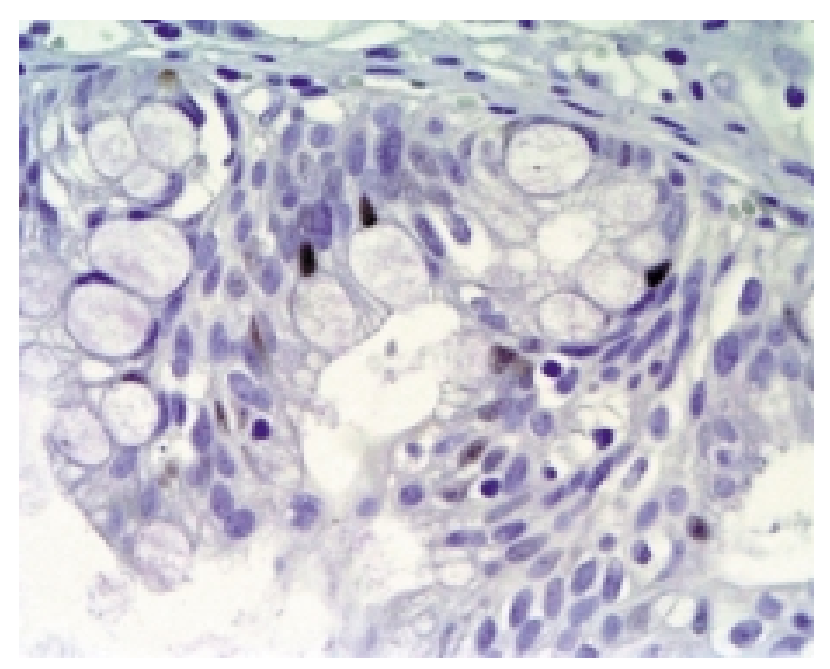

FIGURA 1. Discreta positividad nuclear para CDX2 en las células con morfologia entérica en un caso de cistitis glandularis. (Inmunoperoxidasa, X400). 


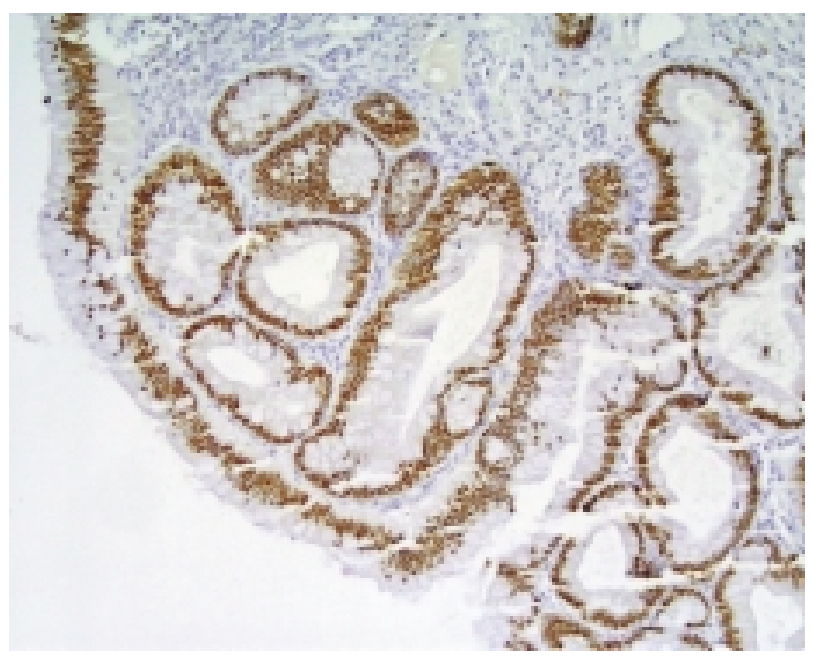

FIGURA 2. Expresión intensa y generalizada de CDX2 en la metaplasia intestinal de vejiga. (Inmunoperoxidasa, X100).

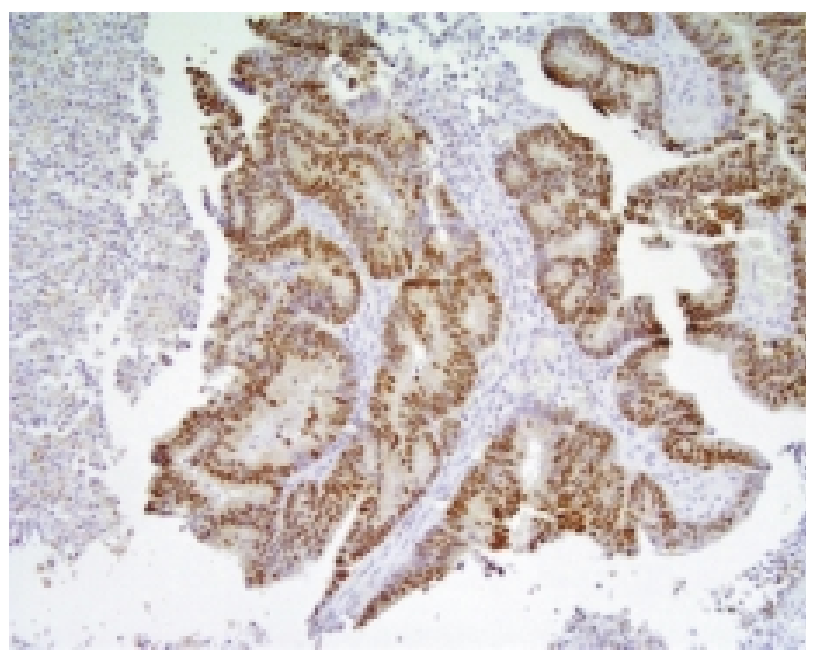

FIGURA 3. Positividad de CDX2 en un adenocarcinoma de tipo intestinal de vejiga. (Inmunoperoxidasa, X200).

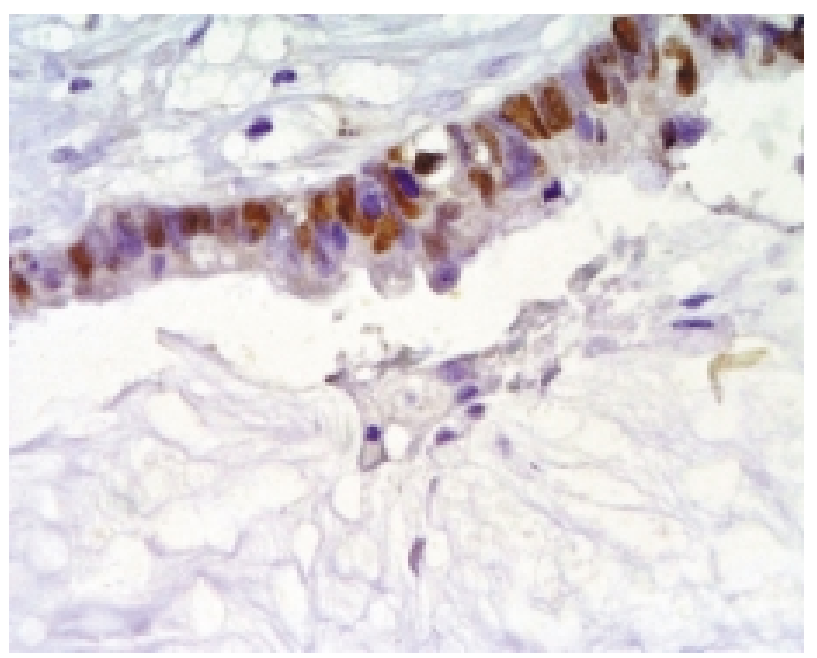

FIGURA 4. Expresión de CDX2 en un carcinoma mucinoso de uraco. (Inmunoperoxidasa, X400).
Fueron negativas para CDX2 las lesiones sin fenotipo de célula entérica, tanto benignas (nidos de Von Brunn, cistitis glandularis típica y adenosis glandular) (Fig. 5), como malignas (carcinoma transicional).

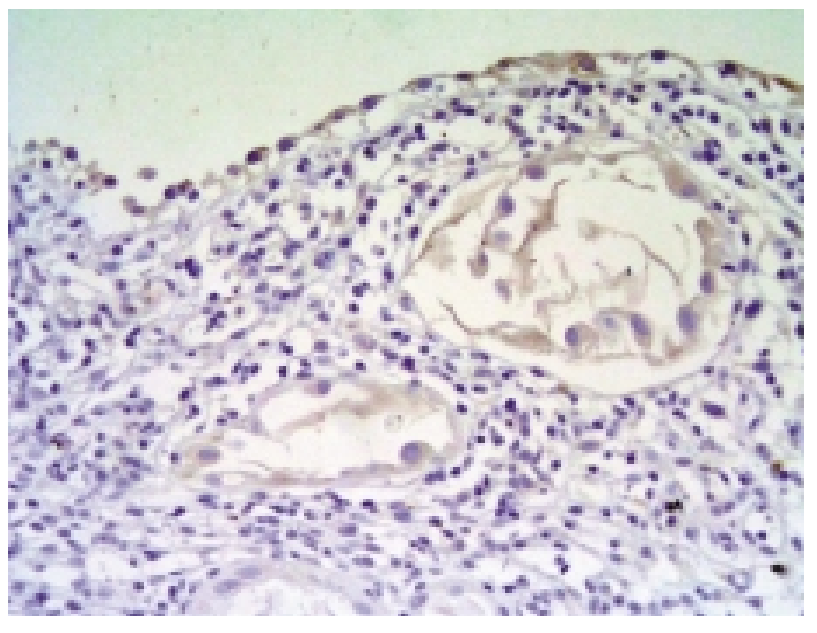

FIGURA 5. Ausencia de inmunorreactividad nuclear para CDX2 en la adenosis glandular. (Inmunoperoxidasa, X200).

\section{DISCUSIÓN}

La expresión de CDX1 y CDX2 constituye un estímulo clave para que se produzca la diferenciación de la célula intestinal normal y se mantenga este fenotipo en el individuo maduro ${ }^{1}$.

Asimismo, la adquisición de un fenotipo intestinal por parte de una célula epitelial de otro tipo, es decir, la metaplasia intestinal, depende de la expresión de CDX1 y CDX2 $2^{1,2,13,20}$ presentando este último un papel esencial en dicho proce$\mathrm{so}^{21,22}$. CDX2 activaría en el núcleo celular una serie de genes, entre los que destaca el de la cadherina-LI, dando lugar, como resultado, a las características morfológicas y funcionales propias de dicho tipo epitelial ${ }^{23,24}$.

La existencia de un epitelio de tipo entérico en la mucosa de la vejiga urinaria se ha descrito en ciertos casos, como en la cistitis glandular de tipo intestinal ${ }^{18}$ y en la metaplasia intestinal, cambio reactivo asociado a situaciones de irritación crónica de la mucosa, debida por ejemplo a sondas, cálculos u obstrucción urinaria ${ }^{14,15}$. La expresión de CDX2 que hemos observado en estas lesiones, confirma el cambio de un epitelio normal a uno de tipo intestinal, con las características moleculares propias del mismo. 
La negatividad para CDX2 en otros cambios reactivos del urotelio, como son los nidos de Von Brunn, la cistitis glandularis típica y la adenosis glandular, demuestra que no guardan relación histogenética con la metaplasia intestinal, la cual se había sugerido en algún caso ${ }^{15}$.

El desarrollo reciente de anticuerpos monoclonales anti-CDX2, ha permitido estudiar su expresión en diferentes neoplasias mediante técnicas de inmunohistoquímica ${ }^{6,8-11}$. Los escasos trabajos realizados se han centrado fundamentalmente en adenocarcinomas de colon y de estómago ${ }^{7,8}$. La expresión de CDX2 se mantiene en la mayor parte de adenomas y adenocarcinomas originados en el epitelio intestinal ${ }^{5}$, con la excepción de aquellos tumores muy poco diferenciados ${ }^{4,8,25}$. Se ha comunicado positividad para CDX2 en un 83,9 a $99 \%$ de adenocarcinomas del colon, según las series ${ }^{7,8,26}$ y en un 28,9 a $70 \%$ de adenocarcinomas gástricos de tipo intestinal ${ }^{7,8}$. Se propuso, por tanto, que CDX2 resultaría un marcador muy sensible y específico de un origen intestinal, o gástrico, en un adenocarcinoma ${ }^{6,7}$. Ello tendría gran utilidad diagnóstica especialmente ante adenocarcinomas metastásicos de origen desconocido o dudoso ${ }^{7,10,11}$.

Se han ido comunicando, sin embargo, casos aislados de adenocarcinomas de otro origen que, al presentar fenotipo de célula intestinal, son positivos para CDX2, como algunos tumores de vesícula o vías biliares ${ }^{7,20}$, adenocarcinomas de esófago $^{7,9}$, páncreas ${ }^{7}$, algunos tumores epiteliales del ovario, sobre todo mucinosos ${ }^{7-10} \mathrm{y}$, muy raramente, adenocarcinoma de endometrio ${ }^{9}$, de pulmón ${ }^{9}$ y de próstata ( $4 \%$ de 27 casos estudiados) ${ }^{7}$.

La expresión de CDX2 en lesiones de la vejiga urinaria o la uretra se ha analizado en muy pocos trabajos ${ }^{7,8}$. En general, el carcinoma transicional es negativo para este marcador, habiéndose encontrado positividad sólo en una serie, en el $2,2 \%$ de 89 tumores $^{8}$, lo que no se confirmó en otros estudios, aunque incluyen un número menor de $\operatorname{casos}^{7}$. Por otra parte, se ha comunicado positividad para CDX2 en dos adenocarcinomas vesicales, de los que no se refiere ningún detalle histológico, y en un carcinoma de uraco ${ }^{7}$.

Los adenocarcinomas del tracto urinario puros, sin componente urotelial, y con histología de tipo intestinal son infrecuentes ${ }^{16,17,19} \mathrm{y}$ proba- blemente se originan sobre un epitelio metaplásico de tipo intestinal preexistente ${ }^{17,27}$. En estos tumores hemos confirmado la expresión de CDX2.

Estos hallazgos implican, en definitiva, que la positividad para este marcador se relaciona con un fenotipo celular y no es órgano-específica. Por ello, en el estudio diagnóstico de tumores metastásicos o de localización pélvica, se debe considerar que la positividad para CDX2 no excluye un posible origen en el tracto urinario.

Por otra parte, comunicamos el segundo caso en la literatura de carcinoma de uraco positivo para CDX2, lo que, una vez más, concuerda con su morfología celular. Este hallazgo se encuentra en la línea de lo observado por Pantuck et al., que demostraron otro epítopo propio del epitelio colónico, detectado mediante el anticuerpo monoclonal $7 \mathrm{E} 12 \mathrm{H} 12$, tanto en el adenocarcinoma de uraco como en el de vejiga, por lo que han sugerido un mecanismo patogénico común para ambas neoplasias ${ }^{27}$.

En conclusión, CDX2, marcador característico de la mucosa del intestino, se expresa en general en el epitelio de tipo entérico que aparece en lesiones benignas de la vejiga (metaplasia intestinal, cistitis glandularis de tipo intestinal) $\mathrm{y}$ en tumores malignos como adenocarcinoma de tipo intestinal de vejiga, carcinoma mucinoso de tipo urotelial de la uretra y adenocarcinoma de uraco. La expresión de CDX2 se encuentra por tanto directamente relacionada con el fenotipo celular y no con el órgano de origen de la lesión.

Agradecimientos. Agradecemos a los Sres. Alfonso Fernández Costas, Eva Peláez Boismorand y $\mathrm{M}^{\mathrm{a}}$ José Barbosa Barreiro su contribución técnica, esencial para la realización de este trabajo.

\section{REFERENCIAS}

1. SILBERG DG, SWAIN GP, RAN SUH E, TRABER PG.: $\mathrm{Cdx} 1$ and Cdx2 expression during intestinal development. Gastroenterology 2000; 119: 961-971.

2. MIZOSHITA T, INADA $K$, TSUKAMOTO $\mathrm{T}$ et al.: Expression of $\mathrm{Cdx} 1$ and $\mathrm{Cdx} 2 \mathrm{mRNAs}$ and relevance of this expression to differentiation in human gastrointestinal mucosa -with special emphasis on participation in intestinal metaplasia of the human stomach. Gastric Cancer 2001; 4: 185-191. 
3. CHAWENGSAKSOPHAK K, JAMES R, HAMMOND VE, KÖNTGEN F, BECK F.: Homeosis and intestinal tumours in Cdx2 mutant mice. Nature 1997; 386: 84-87.

4. MALLO GV, RECHRECHE H, FRIGERIO JM et al.: Molecular cloning, sequencing and expression of the mRNA encoding human $\mathrm{Cdx} 1$ and $\mathrm{Cdx} 2$ homeobox. Down regulation of $\mathrm{Cdx} 1$ and $\mathrm{Cdx} 2 \mathrm{mRNA}$ expression during colorectal carcinogenesis. Int $J$ Cancer 1997; 74: 35-44.

5. GUALTROUGH D, HINOI T, FEARON E, PARASKEVA C.: Expression of CDX2 in normal and neoplastic human colon tissue and during differentiation of an in vitro model system. Gut 2002; 51: 184-190.

6. BARBARESCHI M, MURER B, COLBY TV et al.: CDX-2 homeobox gene expression is a reliable marker of colorectal adenocarcinoma metastases to the lungs. Am J Surg Pathol 2003; 27: 141-149.

7. WERLING RW, YAZIJI H, BACCHI CE, GOWN AM.: CDX2, a highly sensitive and specific marker of adenocarcinomas of intestinal origin. An immunohistochemical survey of 476 primary and metastatic carcinomas. Am J Surg Pathol 2003; 27: 303-310.

8. KAIMAKTCHIEV V, DIRNHOFER S, SAUTER G et al.: Selective staining of gastrointestinal adenocarcinomas by the homeobox intestinal differentiation factor CDX2. Lab Invest 2003; 83: 123A.

9. MAZZIOTA RM, BORCZUK AC, ALEXIS D, MANSUKHANI MM.: Differential expression by immunohistochemistry, of CDX2 transcription factor in various adenocarcinomas. Lab Invest 2003; 83: 127A.

10. GROISMAN GM, MEIR A.: The intestinal marker $\mathrm{Cdx} 2$ is useful in differentiating primary ovarian carcinoma from metastatic colonic carcinoma. Lab Invest 2003; 83: 190A.

11. FURLANETTO A, ORVIETO E, LAURINO L, DEI TOS AP.: Utility of CDX2 immunoreactivity in the diagnosis of metastatic adenocarcinoma to the liver. Lab Invest 2003; 83: 273A.

12. BAI Y-Q, YAMAMOTO H, AKIYAMA Y et al.: Ectopic expression of homeodomain protein CDX2 in intestinal metaplasia and carcinomas of the stomach. Cancer Lett 2002; 176: 47-55.

13. ALMEIDA R, SILVA E, SANTOS-SILVA $\mathrm{F}$ et al.: Expression of intestine-specific transcription factors, CDX1 and CDX2, in intestinal metaplasia and gastric carcinomas. J Pathol 2003; 199: 36-40.

14. LAPERTOSA G, BARACCHINI P, FULCHERI E, TANZI R.: O-acetylated sialic acid variants in intestinal glandular metaplasia of the urinary tract. Histopathology 1986; 10: 707-712.

15. BULLOCK PS, THONI DE, MURPHY WM.: The significance of colonic mucosa (intestinal metaplasia) involving the urinary tract. Cancer 1987; 59: 20862090.

16. TRAN KP, EPSTEIN JI.: Mucinous adenocarcinoma of urinary bladder type arising from the prostatic urethra; distinction from mucinous adenocarcinoma of the prostate. Am J Surg Pathol 1996; 20 (11): 1346-1350.
17. PALMERO MARTÍ JL, QUEIPO ZARAGOZÁ JA, BONILLO GARCÍA MA, BUDÍA ALBA A, VERA SEMPERE FJ, JIMÉNEZ CRUZ JF.: Adenocarcinoma mucinoso de vejiga. Actas Urol Esp 2003; 27: 274280.

18. GUARCH TROYAS R, JIMÉNEZ CALVO J, REPARAZ ROMERO B, GÓMEZ DORRONSORO ML.: Cistitis glandular florida de tipo intestinal con extravasación de mucina: una lesión que simula un tumor. Actas Urol Esp 2003; 27: 297-300.

19. CURTIS MW, EVANS AJ, SRIGLEY JR.: Mucin-producing urothelial-type adenocarcinoma of prostate: a diagnostic challenge addressed by immunohistochemistry. Lab Invest 2003; 83: 146A.

20. REN P, SILBERG DG, SIRICA AE.: Expression of an intestine-specific transcription factor (CDX1) in intestinal metaplasia and in subsequently developed intestinal type of cholangiocarcinoma in rat liver. Am J Pathol 2000; 156: 621-627.

21. EDA A, OSAWA H, YANAKA I et al.: Expression of homeobox gene $\mathrm{CDX} 2$ precedes that of $\mathrm{CDX} 1$ during the progression of intestinal metaplasia. $J$ Gastroenterol 2002; 37: 94-100.

22. MUTOH $\mathrm{H}$, HAKAMATA $\mathrm{Y}$, SATO $\mathrm{K}$ et al.: Conversion of gastric mucosa to intestinal metaplasia in Cdx-2-expressing transgenic mice. Biochem Biophys Res Commun 2002; 294: 470479.

23. FREUND JN, DOMON-DELL C, KEDINGER M et al.: The Cdx-1 and Cdx-2 homeobox genes in the intestine. Biochem Cell Biol 1998; 76: 957-969.

24. HINOI T, LUCAS PC, KUICK R, HANASH S, CHO KR, FEARON ER.: CDX2 regulates liver intestinecadherin expression in normal and malignant colon epithelium and intestinal metaplasia. Gastroenterology 2002; 123: 1565-1577.

25. HINOI T, TANI M, CACA $\mathrm{K}$ et al.: Loss of CDX2 expression and microsatellite instability are prominent features of large cell minimally differentiated carcinomas of the colon. Am J Pathol 2001; 159: 2239-2248.

26. SIVAGNANASUNDARAM S, ISLAM I, TALBOT I et al.: The homeobox gene CDX2 in colorectal carcinoma: a genetic analysis. $B r J$ Cancer 2001; 84: 218-225.

27. PANTUCK AJ, BANCILA E, DAS KM et al.: Adenocarcinoma of the urachus and bladder expresses a unique colonic epithelial epitope: an immunohistochemical study. J Urol 1997; 158: 1722-1727.

Dr. J.A. Ortiz Rey

Servicio de Patología

Centro Médico POVISA

C/ Salamanca, 5

36211 Vigo (Pontevedra)

(Trabajo recibido el 3 noviembre de 2003) 